\title{
Magnetic resonance imaging at a high field strength of ventricular septal defects in infants
}

\author{
E J BAKER, VICTORIA AYTON, * MA SMITH, * J M PARSONS, E J LADUSANS, \\ R H ANDERSON, $\uparrow M$ N MAISEY, $M$ TYNAN, NUALA L K FAGG $\ddagger$ \\ P B DEVERALL $\S$
}

From the Departments of Paediatric Cardiology, ${ }^{*}$ Radiological Sciences; §Cardiothoracic Surgery; and $\ddagger$ Histopathology, Guy's Hospital; and †Cardiothoracic Institute, London

SUMMARY Magnetic resonance imaging at a high field strength has potential benefits for the study of the heart in infants, which is when most congenital heart disease presents. Seventeen infants with various anatomical types of ventricular septal defect were studied by this technique. Good quality, high resolution, images were obtained in every case. There were no major practical problems. The morphology of the defects in all $\mathbf{1 7}$ hearts was displayed in great detail. In some instances, the interpretation of the images resembled that of equivalent images from cross sectional echocardiography. But this new technique allowed imaging in planes that cannot be obtained by echocardiography. One particularly valuable plane gave a face on view of the inlet and trabecular components of the septum. This allowed very precise localisation of defects in these areas. The relation between the defects and the atrioventricular and arterial valves was exceptionally well shown in various different imaging planes. One patient in the series had multiple trabecular defects that were clearly shown.

Magnetic resonance imaging gives detailed morphological information about ventricular septal defects.

High resolution cross sectional imaging can provide a precise description of the morphology of defects in the ventricular septum, ${ }^{1}$ and echocardiography is a useful investigation in patients with such defects. But newer cross sectional imaging methods have not been evaluated in the study of ventricular septal defects. Gated cardiac magnetic resonance imaging, with imaging systems of low field strength, can detect large defects in older patients. ${ }^{23}$ The structural diagnosis of congenital heart defects, however, is usually made in infants." This is the age group in which new imaging techniques need to be evaluated.

We studied an unselected series of infants with various types of ventricular septal defect by a magnetic resonance imaging system that operates at high field strength.

\section{Patients and methods}

\section{PATIENTS}

We studied 17 infants with ventricular septal defects

Requests for reprints to Dr E J Baker, Department of Paediatric Cardiology, Guy's Hospital, London SE1 9RT.

Accepted for publication 10 May 1989 undergoing clinical assessment at Guy's Hospital (table). The selection criteria were the presence of a known ventricular septal defect and the availability of the infant for imaging. They were aged between three weeks and six months. All had a normal atrial arrangement. Nine had normal cardiac connections. In three there was a discordant ventriculoarterial connection, and one of these infants also had a discordant atrioventricular connection. Two cases had double outlet from the morphologically right ventricle, two others had pulmonary atresia, while the final patient had a common arterial trunk. One patient has since died and the heart has been examined (patient 5). Another has undergone surgical closure of the defect (patient 17).

\section{METHODS}

The imaging protocol was described in detail elsewhere. ${ }^{5}$ In all patients an initial series of sections was acquired in the transverse plane of the body, encompassing the whole heart. Typically, this required seven sections. Subsequent imaging planes were chosen in the light of the suspected cardiac abnormality and the position and orientation of the cardiac structures as shown on these,images. Imaging planes 
Table Details of patients studied by magnetic resonance imaging at high field strength

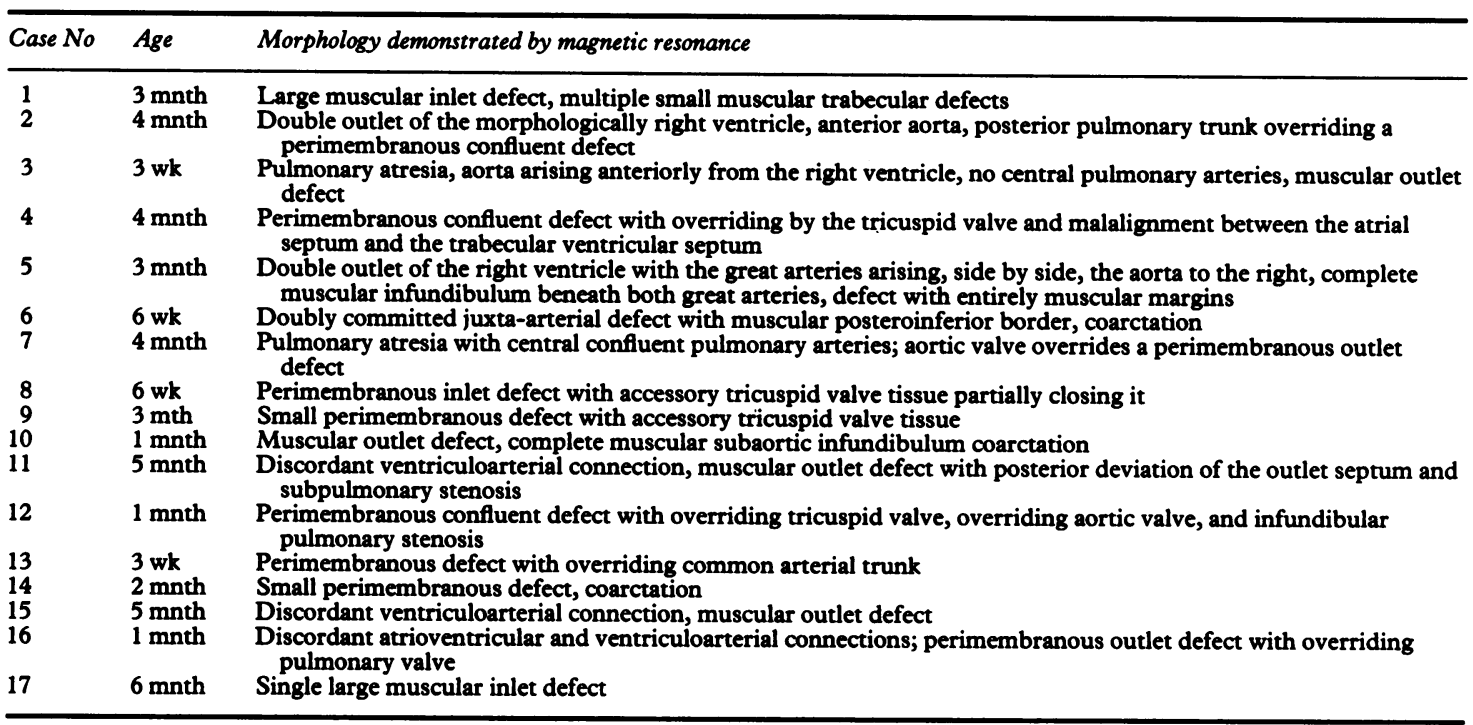

oblique in one or two axes to the standard planes of the body were used in every patient. The angles for these planes were measured individually in each patient from the earlier images. Optimum imaging of the cardiac anomaly was achieved typically in total imaging time of about 90 minutes.

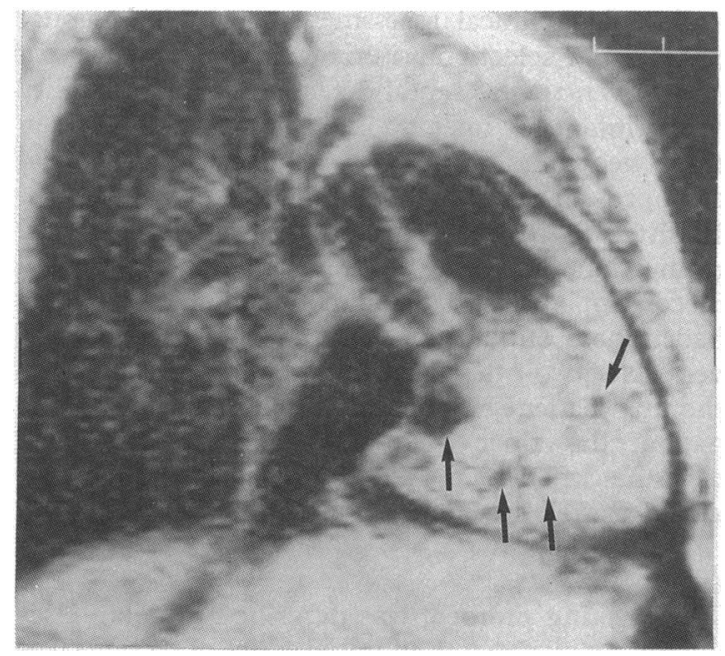

Fig 1 Magnetic resonance image of oblique coronal section in patient 1. The section includes the ventricular septum and shows multiple muscular defects (arrows), the largest being in the inlet septum. There is no defect in the outlet septum; it appears to be defective because it curves backwards out of the plane of the section.

\section{Results}

Images of adequate quality for morphological study were obtained in every patient. The electrocardiographic gating was also satisfactory in all instances.

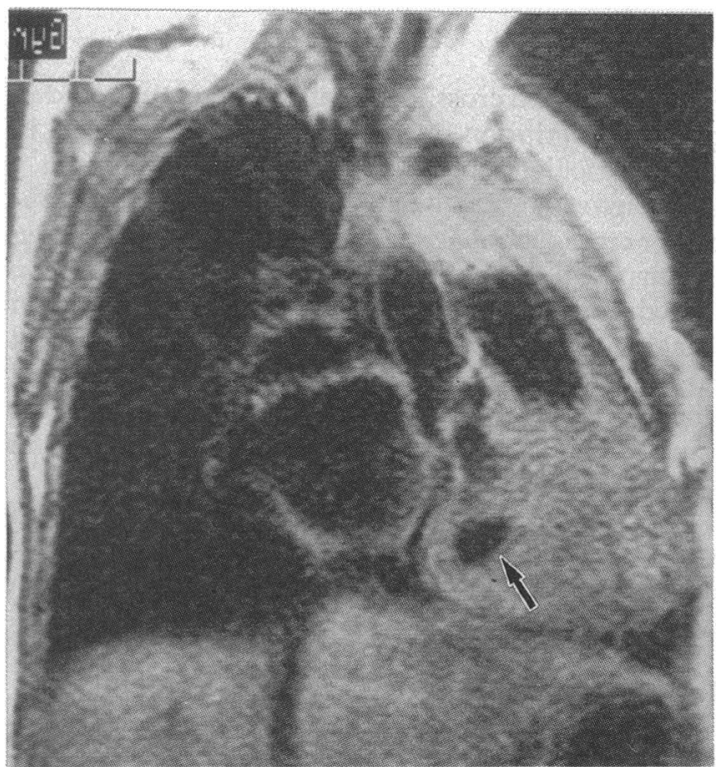

Fig 2 Magnetic resonance image of oblique coronal section in patient 17. There is a single large muscular defect (arrow). The membranous septum was shown to be intact in sections orthogonal to this; because it is thin it appears to be defective in this plane. 


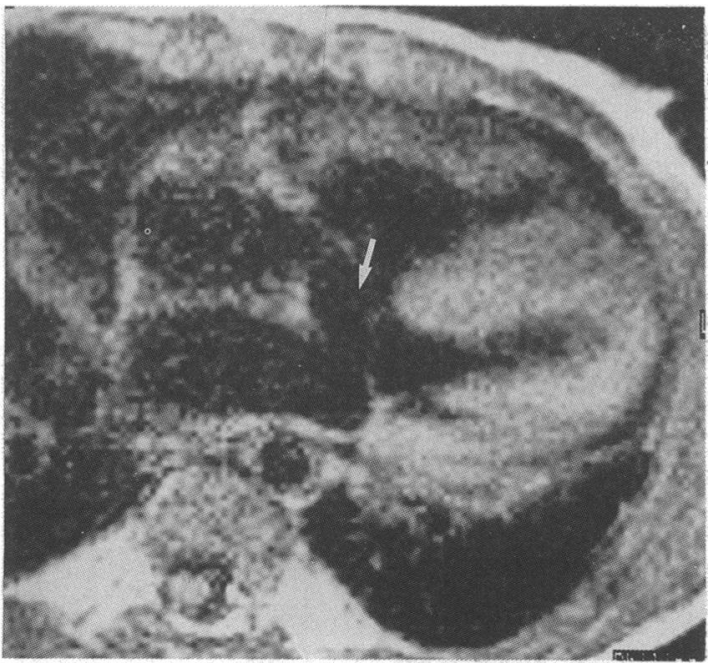

Fig 3 Magnetic resonance image of oblique transverse, four chamber cut, in patient 2 showing that the defect between the ventricular inlets is perimembranous (arrow). The defect is "roofed" by the area of fibrous continuity between the atrioventricular valves, which are not offset.

The table lists the descriptions of the morphology that were discernible from the magnetic resonance images. The morphological criteria for the description of ventricular septal defects have been described in detail elsewhere. ${ }^{1}$ In the two patients in whom direct observation of the morphology of the defect

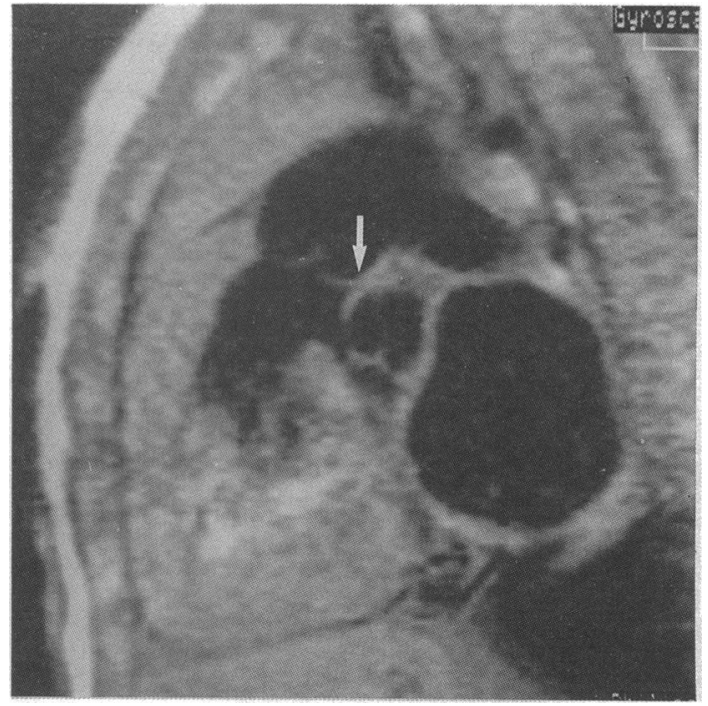

Fig 5 Magnetic resonance image of oblique sagittal cut to show the septum in patient 6 who had a doubly committed juxta-arterial defect. The area of fibrous continuity between the arterial valves is clearly shown (arrow). There is some offsetting between the pulmonary and aortic valves in this section.

was possible at necropsy or operation the magnetic resonance description of the defects was confirmed.

Although the imaging planes used in each patient were different, some general principles emerged. For
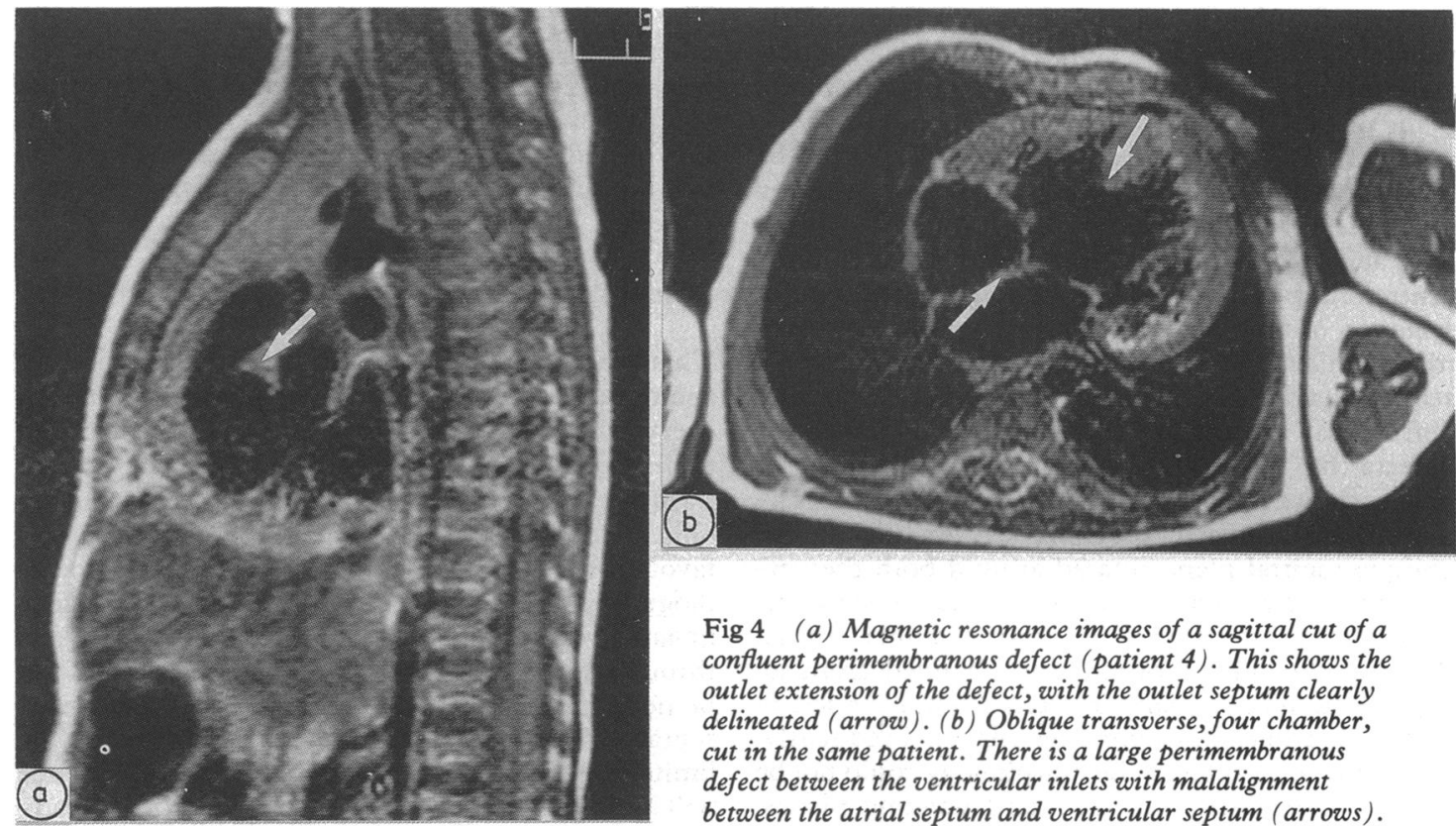

Fig 4 (a) Magnetic resonance images of a sagittal cut of a confluent perimembranous defect (patient 4). This shows the outlet extension of the defect, with the outlet septum clearly delineated (arrow). (b) Oblique transverse, four chamber, cut in the same patient. There is a large perimembranous defect between the ventricular inlets with malalignment between the atrial septum and ventricular septum (arrows). 

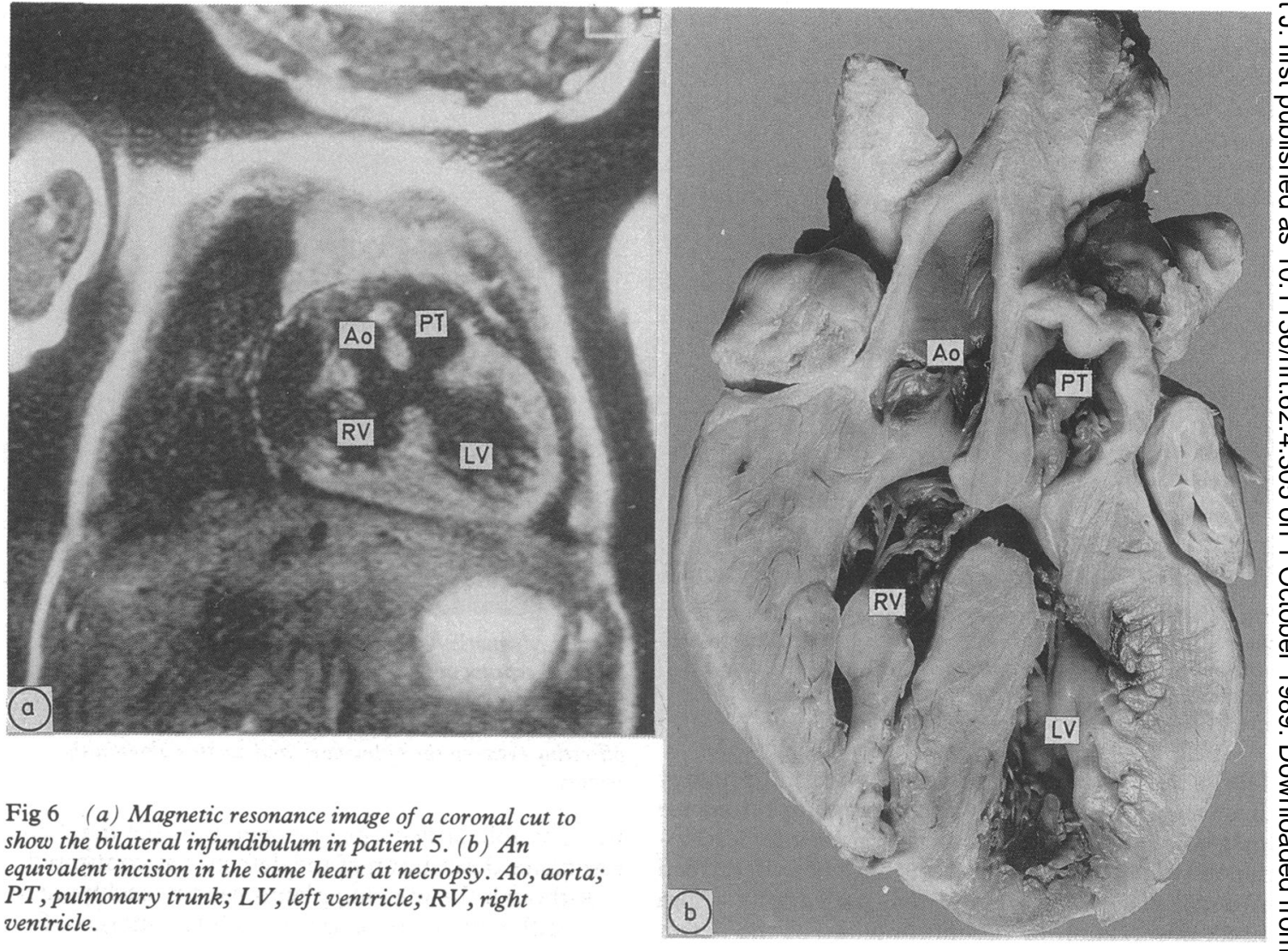

Fig 6 (a) Magnetic resonance image of a coronal cut to show the bilateral infundibulum in patient 5. (b) An equivalent incision in the same heart at necropsy. Ao, aorta; $P T$, pulmonary trunk; $L V$, left ventricle; $R V$, right ventricle.

instance, the oblique coronal plane was a particularly valuable imaging plane (rotated around a vertical axis, and also occasionally a left to right axis, in the plane of the inlet and trabecular components of the ventricular septum). This included a large part of the septum within the $5 \mathrm{~mm}$ thick slice. This imaging plane clearly showed the position of defects in the inlet and trabecular septum and their relation to the central fibrous body. This section needed careful selection of the imaging plane and was not satisfactorily achieved in every patient. In patient 1 , such a section clearly showed not only a large muscular inlet defect, but also several small muscular trabecular defects (fig 1). In contrast, in patient 17 a similar image showed a single large defect with entirely muscular margins in the inlet septum (fig 2). An oblique sagittal plane rotated around both the vertical and anterioposterior axes was used to show the ventricles in short axis. A four chamber image, showing the inlet septum, was obtained from an oblique transverse plane, rotated around the left to right and anterioposterior axes (fig 3). Frequently, even with large defects, the morphology could not be clearly determined from standard imaging planes.
The use of oblique imaging planes individually tailored to the morphology seen in each case was essential (figs 4,5, and 6). The morphology of the ventricular septal defects studied in this series was very varied. There was no standard imaging protocol that would have provided the best images in every patient.

\section{Discussion}

This series contains examples of a wide variety of anatomical types of ventricular septal defects. In $N$ every patient, the magnetic resonance study provided $N$ the morphological details to define the anatomy of $N$ the defects present. The high resolution of the images $\omega$ obtained, even in these small infants, compares favourably with those from cross sectional echocardiography. A unique feature of magnetic resonance $\stackrel{\mathcal{D}}{\rightarrow}$ imaging is its capability to image in any plane through the heart. There should in theory, therefore, $\bar{O}$ be no feature of the morphology of any ventricular $\stackrel{\mathbb{D}}{\circ}$ septal defect that cannot be determined within the $\frac{\vec{D}}{\mathbb{D}}$ limits of resolution of the images. Further experience will indicate whether this is indeed true. It is already 
clear to us that considerable experience is required in choosing the best imaging plane in individual patients. We believe that the standard series of imaging planes that have been described by others, ${ }^{6}$ have no place in the imaging of congenital malformations of the heart. The protocol in each patient has to be matched to the observed anatomy. This is already well established in echocardiography. The same general principles about the interpretation of cross sectional images of the heart apply to magnetic resonance images.

\section{POSITION OF THE DEFECT IN THE VENTRICULAR SEPTUM}

Magnetic resonance images in planes that are equivalent to cross sectional echocardiographic imaging planes can be interpreted by the same criteria. ${ }^{1}$ Thus the defect can be judged as solely opening between the ventricular inlets, solely between the subarterial outlets, or extending between these areas, when they may be termed "confluent" (fig 5). Although there are no examples in this series, large isolated muscular trabecular defects should be demonstrable in such planes. In addition, we showed a new approach to imaging the ventricular septum that is exclusive to the technique of magnetic resonance imaging. Sections in the plane of, and enclosing, the inlet and trabecular components of the septum produce images comparable to seeing these areas of the septum directly. Defects in these areas can then be precisely localised.

RELATION OF THE DEFECT TO ARTERIAL VALVES Examples of muscular, perimembranous, and doubly committed juxta-arterial defects all opening between the subarterial outlets are included in this series. The outlet septum cannot be shown face on in the same way as the inlet and trabecular components of the septum. This is because, in hearts with normal connections, the septum curves posteriorly out of the imaging plane. Despite this, extension of defects to open between the subarterial outlets was exceptionally well shown. For example, the fibrous continuity between aortic and pulmonary valve in a patient with a doubly committed defect was very clear (fig 5). A comparison of the necropsy specimen with the magnetic resonance images in a patient with a bilateral infundibulum shows how well this aspect of the anatomy was shown (fig 6). Features such as overriding of arterial valves and deviation of the outlet septum were also well shown.

RELATION OF THE DEFECT TO THE CENTRAL FIBROUS BODY AND ATRIOVENTRICULAR VALVES The relation of defects to the central fibrous body was shown in various planes. So perimembranous defects were readily distinguished from other defects. Perimembranous defects extending into the inlet septum and roofed by the atrioventricular valves were well shown in a four chamber imaging plane. In two patients in this series such defects were associated with overriding of the tricuspid valve. This was clearly shown (fig 4). In one of these, however, there was echocardiographic evidence of additional straddling of the tension apparatus of the valve that was not evident on the magnetic resonance images.

\section{MULTIPLE DEFECTS}

In one patient in this series, the magnetic resonance images clearly showed multiple small trabecular defects in association with a large muscular defect of the inlet septum (fig 1). Only one trabecular defect was shown in this patient on both Doppler colour flow mapping and cineangiography. It is, therefore, possible that magnetic resonance imaging will prove of special value in detecting the presence of multiple defects. Great care is needed to differentiate trabeculations on the right ventricular surface of the septum from true small trabecular defects. By following the defects in contiguous sections from the cavity of the left ventricle to that of the right we were confident that in this patient they were true septal defects. If this is not always done, trabeculations will be falsely identified as septal defects.

THE PLACE OF MAGNETIC RESONANCE IMAGING There are few practical problems with this imaging technique. The patient must be prevented from moving. We found that light sedation was sufficient for this. The infant does not suffer any discomfort during the imaging procedure and, if well prepared, will generally sleep soundly. The heart rate is constantly monitored and the patient's colour and respiratory motion can be observed from outside the magnet. Cardiac gating was a potential problem because of possible distortion of the electrocardiogram. But we have not found any major difficulty with this. Occasionally the position of the electrodes has to be adjusted to give the best signal, but we have always been able to obtain satisfactorily gating.

Magnetic resonance imaging can, therefore, provide excellent images of ventricular septal defects. Much of the information they contain is comparable with that from cross sectional echocardiography. The images, however, are of high resolution, and new imaging planes that are not possible with echocardiography can be used. This new technique enables a very detailed assessment of the morphology of ventricular septal defects to be made non-invasively. This calls into question the role of 
cineangiography in the assessment of infants with ventricular septum defects.

This research was supported by the British Heart Foundation. The imaging system was purchased with the assistance of Sir Philip and Lady Harris and the Special Trustees of Guy's Hospital.

\section{References}

1 Baker EJ, Leung MP, Anderson RH, Fischer DR, Zuberbuhler JR. The cross sectional anatomy of ventricular septal defects: a reappraisal. $B r$ Heart $J$ 1988;59:339-51.

2 Didier D, Higgins CB, Fischer MR, Osaki L, Silverman
$\mathrm{NH}$, Cheitlin MD. Congenital heart disease: gated MR imaging in 72 patients. Radiology 1986;158: 227-35.

3 Underwood SR, Firmin DN, Klipstein RH, Rees RSO, Longmore DB. Magnetic resonance velocity mapping: clinical application of a new technique. Br Heart $J$ 1987;57:404-12.

4 Hoffman JIE, Christianson R. Congenital heart disease in a cohort of 19,502 births with long term follow up. Am J Cardiol 1978;42:641-7.

5 Baker EJ, Ayton V, Smith MA, et al. Magnetic resonance imaging of coarctation of the aorta in infants: use of a high field strength. Br Heart J 1989;62:97-101.

6 Didier D, Higgins CB. Identification and localisation of ventricular septal defects by gated magnetic resonance imaging. Am J Cardiol 1986;57:1363-8. 